\title{
Achieving Energy Efficiency Fairness in Multicell MISO Downlink
}

\author{
Kien-Giang Nguyen, Le-Nam Tran, Oskari Tervo, Quang-Doanh Vu, and Markku Juntti
}

\begin{abstract}
We investigate the fairness of achievable energy efficiency in a multicell multiuser multiple-input single-output (MISO) downlink system, where a beamforming scheme is designed to maximize the minimum energy efficiency among all base stations. The resulting optimization problem is a nonconvex max-min fractional program, which is generally difficult to solve optimally. We propose an iterative beamformer design based on an inner approximation algorithm which aims at locating a Karush-Kuhn-Tucker solution to the nonconvex program. By novel transformations, we arrive at a convex problem at each iteration of the proposed algorithm, which is amendable for being approximated by a second order cone program. The numerical results demonstrate that the proposed algorithm outperforms the existing schemes in terms of the convergence rate and processing time.
\end{abstract}

Index Terms-Energy efficiency, max-min fractional programming, inner approximation algorithm.

\section{INTRODUCTION}

Multi-antenna technologies have been a key element to increase the capacity of wireless communications. Traditionally, design criteria for communications systems have mainly concerned with spectral efficiency maximization or sum power minimization. Recently, energy efficiency (EE) has emerged as an important criterion to address a growing concern over the global climate and sustainable economic growth.

Decreasing cell sizes and employing small-cell networks are potential solutions to the problem of capacity crunch in future cellular networks. However, this may cause the transceiver processing power to be a significant portion of the total power budget. Taking this additional power into account, energy-efficient approaches have been investigated in multipleinput multiple-output (MIMO) systems, by optimizing the EE subject to sum power and quality of service constraints [1]. Another important problem is to maintain the fairness of the achievable EE among involving parties. To do so, a widely used method is to maximize the minimum EE of the system, which was studied in, e.g., [2], [3], [4].

This work was supported in part by the Finnish Funding Agency for Innovation (Tekes) under the project "Cross-Layer Modeling and Design of Energy-Aware Cognitive Radio Networks (CREAM)" and the Academy of Finland under project "WiFiUS: Message and CSI Sharing for Cellular Interference Management with Backhaul Constraints".

K.-G. Nguyen, O. Tervo and M. Juntti are with the Department of Communications Engineering and Centre for Wireless Communications, University of Oulu, FI-90014, Finland (e-mail: \{nguyen.k.giang, oskarite, markku.juntti\}@ee.oulu.fi).

L.-N. Tran is with the Department of Electronic Engineering, Maynooth University, Ireland (e-mail: ltran@eeng.nuim.ie).

Q.-D. Vu is with the School of Electronics and Information, Kyung Hee University, Yongin 449-701, South Korea (e-mail: vqdoanh@khu.ac.kr).
The problem encountered in max-min EE is basically a max-min fractional program which is nonconvex and, thus, generally difficult to solve to global optimum [5]. In [2], maxmin EE among base stations (BSs) with total transmit power and signal-to-interference-plus-noise ratio (SINR) constraints was studied for single-input single-output interference channels. In [3], max-min EE among users for multiuser multipleinput single-output (MISO) channels was considered. Both of these use Dinkelbach (DB) type algorithms, requiring twolayer iterative procedures to yield a solution. This may result in a relatively large number of iterations to solve the problem.

In this letter, we consider the problem of max-min EE among BSs with BS-specific power constraints in multicell MISO downlink, and propose an iterative beamformer design to solve this problem. The proposed design is based on an inner approximation algorithm originally introduced in [6], which aims at locating the Karush-Kuhn-Tucker (KKT) solution to the nonconvex program. Furthermore, exploiting the structure of the convex problem that needs to be solved at each iteration and invoking an interesting result in [7], we are able to approximate it as a second order cone program (SOCP). This helps to reduce the overall runtime of the proposed algorithm thanks to extremely efficient state-ofthe-art SOCP solvers. The numerical results show that the proposed algorithm greatly improves the convergence rate and processing time compared to the existing schemes. It is worth mentioning that sequential convex programming (similar to inner approximation method) was used to study the problem of energy-efficient power control in [4].

\section{System Model ANd Problem Formulation}

We consider a $B$-cell MISO downlink system where each cell consists of a single BS equipped with $N$ antennas. The BS $b, b \in \mathcal{B}=\{1, \ldots, B\}$, is assumed to serve $K_{b}$ single-antenna users in its own cell. The set of users receiving the data from BS $b$ is denoted by $\mathcal{K}_{b}=\left\{1, \ldots, K_{b}\right\}$. We define the $k$ th user of cell $b$ as $b_{k}$ for $b \in \mathcal{B}$ and $k \in \mathcal{K}_{b}$. The received signal at user $b_{k}$ can be written as

$$
y_{b_{k}}=\sum_{j=1}^{K_{b}} \mathbf{h}_{b, b_{k}} \mathbf{w}_{b_{j}} s_{b_{j}}+\sum_{i=1, i \neq b}^{B} \sum_{j=1}^{K_{i}} \mathbf{h}_{i, b_{k}} \mathbf{w}_{i_{j}} s_{i_{j}}+n_{b_{k}}
$$

where $\mathbf{h}_{i, b_{k}} \in \mathbb{C}^{1 \times N}$ is the channel vector from BS $i$ to user $b_{k}, \mathbf{w}_{b_{k}} \in \mathbb{C}^{N \times 1}$ and $s_{b_{k}}$ are the beamforming vector and the transmit data symbol from BS $b$ to user $b_{k}$, respectively, and $n_{b_{k}} \sim \mathcal{C N}\left(0, \sigma_{b_{k}}^{2}\right)$ is the additive white Gaussian noise with variance $\sigma_{b_{k}}^{2}$. We can write the SINR for user $b_{k}$ as

$$
\gamma_{b_{k}}(\mathbf{w})=\left|\mathbf{h}_{b, b_{k}} \mathbf{w}_{b_{k}}\right|^{2} /\left(I_{b_{k}}(\mathbf{w})+\sigma_{b_{k}}^{2}\right)
$$


where $\mathbf{w}$ is a vector encompassing the beamformers of all users, and $I_{b_{k}}(\mathbf{w}) \triangleq \sum_{j=1, j \neq k}^{K_{b}}\left|\mathbf{h}_{b, b_{k}} \mathbf{w}_{b_{j}}\right|^{2}+$ $\sum_{i=1, i \neq b}^{B} \sum_{j=1}^{K_{i}}\left|\mathbf{h}_{i, b_{k}} \mathbf{w}_{i_{j}}\right|^{2}$ is the interference to user $b_{k}$, which is treated as Gaussian noise. Then, the EE of BS $b$ can be expressed as

$$
f_{b}(\mathbf{w})=\frac{\sum_{k=1}^{K_{b}} \log \left(1+\gamma_{b_{k}}(\mathbf{w})\right)}{\frac{1}{\epsilon} \sum_{k=1}^{K_{b}}\left\|\mathbf{w}_{b_{k}}\right\|_{2}^{2}+N P_{\mathrm{dp}}+P_{\mathrm{sp}}}
$$

where $\epsilon$ and $P_{0} \triangleq N P_{\mathrm{dp}}+P_{\mathrm{sp}}$ are the power amplifier efficiency and the total circuit power, respectively, $P_{\mathrm{dp}}$ is the dynamic power consumption corresponding to the power radiation of all circuit blocks in each active radio frequency chain, and $P_{\mathrm{sp}}$ is the static power spent by the cooling system, power supply, etc. [8]. We are interested in the problem of maximizing the minimum $\mathrm{EE}$ to achieve the $\mathrm{EE}$ fairness among all the BSs, which is mathematically stated as

$$
\max _{\mathbf{w}} \min _{b \in \mathcal{B}}\left\{f_{b}(\mathbf{w}) \mid \sum_{k=1}^{K_{b}}\left\|\mathbf{w}_{b_{k}}\right\|_{2}^{2} \leq P_{b}\right\}
$$

where $P_{b}$ is the transmit power budget of BS $b$.

\section{Proposed Low-COMPLEXITY BEAMFORMING DESIGN}

Problem (4) is a max-min fractional program, for which a common approach is based on solving a parametric program and a two-layer iterative procedure, i.e., a Dinkelbach-type algorithm [9], [10]. As numerically shown in Sect. IV, applying a Dinkelbach-type method such as the one in [3] to problem (4) results in a slow convergence rate. To address this issue, we propose a one-layer iterative algorithm based on an inner approximation framework. Let us start by rewriting (4) equivalently as

$$
\begin{aligned}
& \max _{\mathbf{w}, \eta, \mathbf{z}, \mathbf{t}, \mathbf{g}} \eta \\
& \text { subject to } z_{b}^{2} / t_{b} \geq \eta, \forall b \in \mathcal{B} \\
& \sum_{k=1}^{K_{b}} \log \left(1+g_{b_{k}}\right) \geq z_{b}^{2}, \forall b \in \mathcal{B} \\
& \left|\mathbf{h}_{b, b_{k}} \mathbf{w}_{b_{k}}\right|^{2} /\left(I_{b_{k}}(\mathbf{w})+\sigma_{b_{k}}^{2}\right) \geq g_{b_{k}} \\
& \frac{1}{\epsilon} \sum_{k=1}^{K_{b}}\left\|\mathbf{w}_{b_{k}}\right\|_{2}^{2}+P_{0} \leq t_{b} \forall b \in \mathcal{B} \\
& \sum_{k=1}^{K_{b}}\left\|\mathbf{w}_{b_{k}}\right\|_{2}^{2} \leq P_{b}, \forall b \in \mathcal{B}
\end{aligned}
$$

where $\eta, \mathbf{z} \triangleq\left\{z_{b}\right\}_{b \in \mathcal{B}}, \mathbf{t} \triangleq\left\{t_{b}\right\}_{b \in \mathcal{B}}$ and $\mathbf{g} \triangleq\left\{g_{b_{k}}\right\}_{b \in \mathcal{B}, k \in \mathcal{K}_{b}}$ are newly introduced variables. The equivalence between (4) and (5) follows from the fact that the constraints from (5b) to (5e) hold with equality at optimality, which can be proved using the same arguments as those in [11]. In the same spirit, we further split (5d) into two constraints as

$$
\begin{aligned}
\left|\mathbf{h}_{b, b_{k}} \mathbf{w}_{b_{k}}\right|^{2} / q_{b_{k}} & \geq g_{b_{k}} \\
q_{b_{k}} & \geq I_{b_{k}}(\mathbf{w})+\sigma_{b_{k}}^{2}
\end{aligned}
$$

where $q_{b_{k}} \in \mathbf{q} \triangleq\left\{q_{b_{k}}\right\}_{b \in \mathcal{B}, k \in \mathcal{K}_{b}}$ is the newly introduced variable, which can be interpreted as the soft interference at user $b_{k}$. In summary, we have arrived at an equivalent formulation of (4) given by

$$
\begin{array}{ll}
\max _{\mathbf{w}, \eta, \mathbf{z}, \mathbf{t}, \mathbf{g}, \mathbf{q}} & \eta \\
\text { subject to } & z_{b}^{2} / t_{b} \geq \eta, \quad \forall b \in \mathcal{B}
\end{array}
$$

$$
\begin{aligned}
& \left|\mathbf{h}_{b, b_{k}} \mathbf{w}_{b_{k}}\right|^{2} / q_{b_{k}} \geq g_{b_{k}}, \quad \forall b \in \mathcal{B}, k \in \mathcal{K}_{b} \\
& \text { (5c),(5e),(5f),(6b). }
\end{aligned}
$$

It is now clear that the difficulty in solving (7) is due to (7b) and $(7 \mathrm{c})$ since the remaining constraints in (7) are convex. We remark that (7b) and (7c) are of the same type where the right side of the constraint is an affine function and the left side is a quadratic-over-affine function, which is jointly convex w.r.t. the involved variables. Let us focus on the constraints in (7b) first. In particular, due to the convexity of $z_{b}^{2} / t_{b}$, we have

$$
z_{b}^{2} / t_{b} \geq 2\left(z_{b}^{(n)} / t_{b}^{(n)}\right) z_{b}-\left(z_{b}^{(n)} / t_{b}^{(n)}\right)^{2} t_{b} \triangleq \phi_{b}^{(n)}\left(z_{b}, t_{b}\right)
$$

where the superscript $n$ denotes the $n$th iteration of the iterative algorithm presented shortly. Note that $\phi_{b}^{(n)}\left(z_{b}, t_{b}\right)$ is in fact the first order of $z_{b}^{2} / t_{b}$ around the point $\left(z_{b}^{(n)}, t_{b}^{(n)}\right)$. In light of [6], we can iteratively replace $z_{b}^{2} / t_{b}$ in (7b) by $\phi_{b}^{(n)}\left(z_{b}, t_{b}\right)$ to achieve a convex approximation of $(7 \mathrm{~b})$. Similarly, we can approximate $(7 \mathrm{c})$ by the linear constraint $\psi_{b_{k}}^{(n)}\left(\mathbf{w}_{b_{k}}, q_{b_{k}}\right) \geq g_{b_{k}}$ where $\psi_{b_{k}}^{(n)}\left(\mathbf{w}_{b_{k}}, q_{b_{k}}\right)$ is the first order of $\left|\mathbf{h}_{b, b_{k}} \mathbf{w}_{b_{k}}\right|^{2} / q_{b_{k}}$ around $\mathbf{w}_{b_{k}}^{(n)}$ and $q_{b_{k}}^{(n)}$, which is given by

$$
\begin{aligned}
\psi_{b_{k}}^{(n)}\left(\mathbf{w}_{b_{k}}, q_{b_{k}}\right) \triangleq & 2 \Re\left(\left(\mathbf{w}_{b_{k}}^{(n)}\right)^{H} \mathbf{h}_{b, b_{k}}^{H} \mathbf{h}_{b, b_{k}} \mathbf{w}_{b_{k}}\right) / q_{b_{k}}^{(n)} \\
& -\left(\left|\mathbf{h}_{b, b_{k}} \mathbf{w}_{b_{k}}^{(n)}\right| / q_{b_{k}}^{(n)}\right)^{2} q_{b_{k}} .
\end{aligned}
$$

From the above discussions, the approximate convex problem solved at iteration $n+1$ of the proposed design is given by

$$
\begin{array}{cl}
\max _{\mathbf{w}, \eta, \mathbf{z}, \mathbf{t}, \mathbf{g}, \mathbf{q}} & \eta \\
\text { subject to } & \phi_{b}^{(n)}\left(z_{b}, t_{b}\right) \geq \eta, \forall b \in \mathcal{B} \\
& \psi_{b_{k}}^{(n)}\left(\mathbf{w}_{b_{k}}, q_{b_{k}}\right) \geq g_{b_{k}}, \forall b \in \mathcal{B}, k \in \mathcal{K}_{b} \\
& (5 \mathrm{c}),(5 \mathrm{e}),(5 \mathrm{f}),(6 \mathrm{~b}) .
\end{array}
$$

After solving (10), we update the involved variables for the next iteration until convergence. Algorithm 1 outlines the proposed iterative algorithm for solving the considered problem. In Algorithm 1, we generate initial points $\left(\mathbf{w}^{(0)}, \mathbf{z}^{(0)}, \mathbf{t}^{(0)}, \mathbf{q}^{(0)}\right)$ as follows. First, a set of beamformers that satisfy (5f) is created. Then the initial values of other variables are obtained by setting all the constrains in (7) to be equality. In this way, Algorithm 1 always starts with a feasible solution of (7).

Convergence analysis: It is readily seen that the optimal solutions returned at the iteration $n$ are also feasible for the problem at the iteration $n+1$, which is due to the approximations in (10b) and (10c) [6]. This implies that Algorithm 1 yields a nondecreasing sequence of the objective, i.e., $\eta^{(n+1)} \geq \eta^{(n)}$. It is easy to see that the sequence $\left\{\eta^{(n)}\right\}$ is bounded above due to the power constraints in (5f). Thus, Algorithm 1 is guaranteed to converge. Following the same arguments as those in [6], we can prove that the proposed algorithm converges to a KKT solution of (7).

\section{Solving (10) using a conic programming solver}

We note that the formulation given in (10) is classified as a generic convex program (GCP). Although it can be solved efficiently by a modern convex solver such as MOSEK [12], it generally requires more computation time, compared to other standard convex programs such as the second order cone 


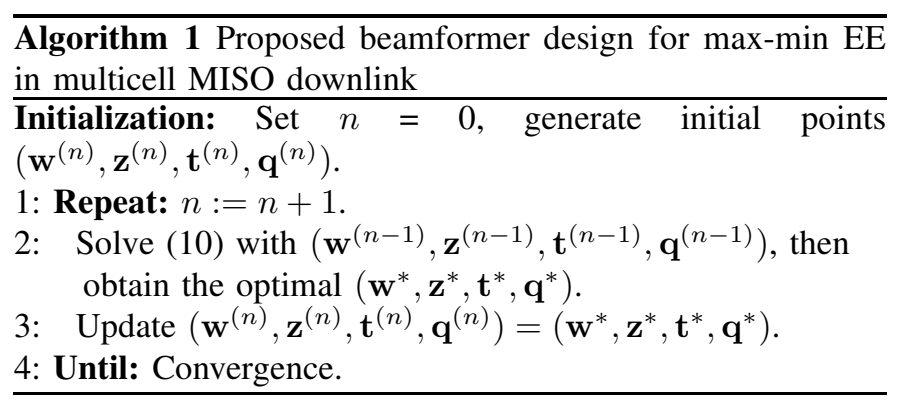

programming. Exploiting the specific structure of (10), we are able to show that it can be approximated as an SOCP to a desired accuracy. Towards this end, we remark that all the constraints in (10), excluding (5c), are linear or SOC representable (see [13, Sect. 3.3] for further details). To see that $(5 \mathrm{c})$ can be approximated as SOCs, we first rewrite $(5 \mathrm{c})$

$$
\begin{aligned}
\sum_{k=1}^{K_{b}} \beta_{b_{k}} & \geq z_{b}^{2} \\
1+g_{b_{k}} & \geq e^{\beta_{b_{k}}} .
\end{aligned}
$$

where $\beta_{b_{k}}$ is newly introduced variable. Again note that (11) is SOC representable [13, Sect. 3.3]. A result in [7] states that (12) can be approximated by a system of conic constraints as

$$
\begin{aligned}
& 1+g_{b_{k}} \geq \kappa_{0} \\
& 1+\kappa_{1} \geq\left\|\left[\begin{array}{ll}
1-\kappa_{1} & 2+\beta_{b_{k}} / 2^{m-1}
\end{array}\right]\right\|_{2} \\
& 1+\kappa_{2} \geq\left\|\left[\begin{array}{ll}
1-\kappa_{2} & 5 / 3+\beta_{b_{k}} / 2^{m}
\end{array}\right]\right\|_{2} \\
& 1+\kappa_{3} \geq\left\|\left[\begin{array}{ll}
1-\kappa_{3} & 2 \kappa_{1}
\end{array}\right]\right\|_{2} \\
& \kappa_{4} \geq \kappa_{2}+\kappa_{3} / 24+19 / 72 \\
& 1+\kappa_{l} \geq\left\|\left[\begin{array}{ll}
1-\kappa_{l} & 2 \kappa_{l-1}
\end{array}\right]\right\|_{2}, l \in\{5, \ldots, m+3\} \\
& 1+\kappa_{0} \geq\left\|\left[\begin{array}{ll}
1-\kappa_{0} & 2 \kappa_{m+3}
\end{array}\right]\right\|_{2}
\end{aligned}
$$

where $\kappa_{l}, l=0,1, \ldots, m+3$, are newly introduced variables, and the accuracy of the approximation increases with $m$. Assuming zero-interference, we can bound $\beta_{b_{k}}$ as $0 \leq \beta_{b_{k}} \leq$ $\log \left(1+\left|\mathbf{h}_{b, b_{k}} \mathbf{w}_{b_{k}}\right|^{2} / \sigma_{b_{k}}^{2}\right) \leq \log \left(1+P_{b}|| \mathbf{h}_{b, b_{k}} \|_{2}^{2} / \sigma_{b_{k}}^{2}\right)=$ $\bar{\beta}_{b_{k}}$. For a given accuracy $\varepsilon$, the value of $m$ is given by $m=\mathcal{O}\left(\ln \frac{\bar{\beta}_{b_{k}}}{\varepsilon}\right)$ to approximate (12) by (13) [7]. Invoking (13), we can approximate the problem solved at the iteration $n+1$ of the proposed algorithm as an SOCP given by $\max \{\eta \mid(5 e),(5 f),(6 b),(10 b),(10 c),(11),(13)\}$. We have numerically observed that the error between the objectives of (10) and the approximate SOCP is smaller than $10^{-5}$ when $m=10$.

Complexity analysis: We now provide worst-case periteration complexity analysis of Algorithm 1 using (12) and (13), following the results in [13, Sect. 6.6]. Specifically, the per-iteration cost of solving the GCP using (12) and the SOCP using (13) is $\mathcal{O}\left(N^{4} K^{4}+B^{4}\right)$ and $\mathcal{O}\left(N^{3} K^{3}+B^{3}+(m+\right.$ $7)^{3} K^{3}$ ), respectively, where $K=\sum_{b=1}^{B} K_{b}$ is the total number of users. We see that introducing slack variables increases the per-iteration complexity of Algorithm 1, compared to the iterative procedure in [3] which iteratively requires solving an SOCP with per-iteration complexity of $\mathcal{O}\left(N^{3} K^{3}\right)$. Note that these complexity estimates are typically conservative in practice. More importantly, the overall runtime of Algorithm 1 and the iterative algorithm in [3] largely depends on the actual number of iterations to converge, which is hard to
Table I

SIMULATION PARAMETERS

\begin{tabular}{c|c}
\hline PARAMETERS & VALUE \\
\hline \hline Pathloss and shadowing & $38 \log (d)+34.5+\mathcal{N}(0,8)[\mathrm{dB}]$ \\
Cell radius & $500[\mathrm{~m}]$ \\
Transmit power constraint $P_{b}$ & $35[\mathrm{dBm}]$ \\
Static power consumption $P_{\mathrm{sp}}$ & $33[\mathrm{dBm}]$ \\
Power amplifier efficiency $\epsilon$ & 0.35 \\
Number of BSs $B$ & 3 \\
Signal bandwidth & $10[\mathrm{kHz}]$ \\
Power spectral density of noise & $-174[\mathrm{dBm} / \mathrm{Hz}]$ \\
\hline
\end{tabular}

analytically predict. The numerical comparison of convergence rate provided in Figs. 2 and 3 shows that the proposed algorithm converges remarkably fast, and, thus, needs much less runtime than the one in [3]. Further discussions on the complexity comparison are given in the next section.

\section{NUMERICAL RESULTS}

In this section, we demonstrate the effectiveness of the proposed method by numerical experiments. The simulation parameters are listed in Table I. All the convex problems considered in this paper are solved using the MOSEK solver in MATLAB environment.

In particular, we compare the proposed design with the iterative method introduced in [3] which is referred to as the WMMSE-DB method. Basically, the WMMSE-DB method is based on an alternating optimization framework where an SOCP is solved at each iteration (see [3] for further details). The initial beamformers $\mathbf{w}^{(0)}$ in Algorithm 1 are also used to start the WMMSE-DB method. All iterative algorithms of comparison are terminated if the increase of the objective between two consecutive iterations is smaller than $10^{-5}$.

Fig. 1 plots the average runtime of Algorithm 1 and the WMMSE-DB method as a function of the total number of users. As can be seen, the proposed algorithm outperforms the method of [3], especially when using the conic approximation in (13). The main reason is that the proposed algorithm converges much faster than the WMMSE-DB method as we show in Figs. 2 and 3. Particularly, the conic approximation offers significant reduction of runtime due to the fact that an SOCP can be solved much more efficiently, compared to a GCP of the similar size. We remark that SOCPs are wellstructured convex problems which make them easier to solve [13, Sect. 6.2].

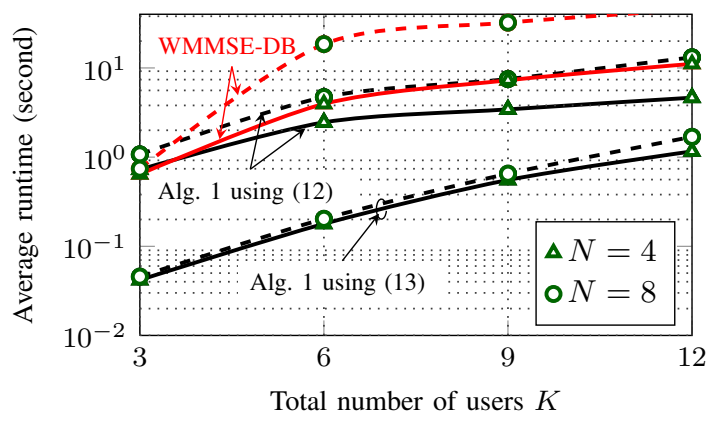

Fig. 1. Runtime comparison of different algorithms for max-min EE fairness with $P_{\mathrm{dp}}=40 \mathrm{dBm}$. 


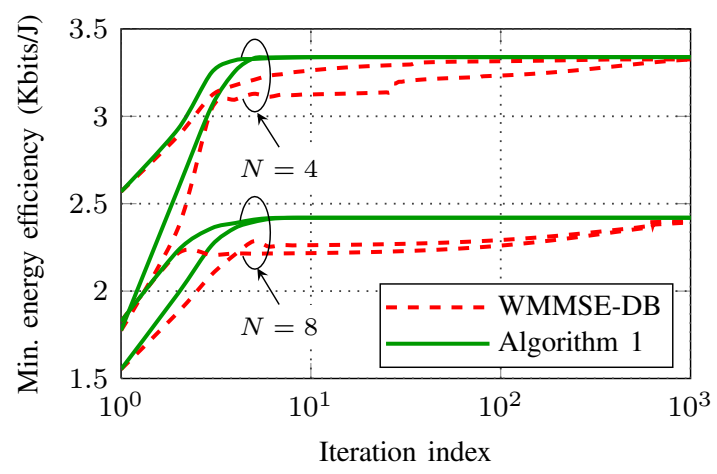

Fig. 2. Convergence of the algorithms with $K_{b}=2$ and $P_{\mathrm{dp}}=40 \mathrm{dBm}$.

Fig. 2 compares the convergence speed of Algorithm 1 and the WMMSE-DB method for a randomly generated channel realization from two different initial points. We can see that both methods converge to the same objective value. However, the proposed algorithm converges after a few iterations, while it takes hundreds of iterations for the WMMSE-DB method to stabilize. Recall that the WMMSE-DB method is based on an alternating optimization framework, in which some of the variables are updated at each iteration. In the proposed method, all optimization variables are accounted for to find a better solution for the next iteration.

To achieve a more complete comparison of convergence rate of Algorithm 1 and the WMMSE-DB method, we plot the cumulative distribution function (CDF) of the number of iterations in Fig. 3. As can be seen, for $90 \%$ of the channel realizations, the proposed method converges after 20 iterations, while it can be thousands of iterations for the WMMSE-DB scheme. Another observation is that as $P_{\mathrm{dp}}$ decreases, both methods of comparison require more iterations to converge. We note that the variables satisfying $(5 \mathrm{e})$ for large $P_{\mathrm{dp}}$ (i.e., large $P_{0}$ ) also satisfy it for smaller $P_{\mathrm{dp}}$, meaning that the feasible set of (5) becomes larger when $P_{\mathrm{dp}}$ is smaller. Thus, more iterations are generally needed for a larger feasible set.

Fig. 4 illustrates the average EE versus the dynamic power consumption $P_{\mathrm{dp}}$ for all the BSs with the proposed design. We have observed that for some channel realizations, the three BSs do not attain the same EE when the proposed design converges. However, on an average sense, all BSs achieve almost the same performance as shown in Fig. 4. This basically implies that the

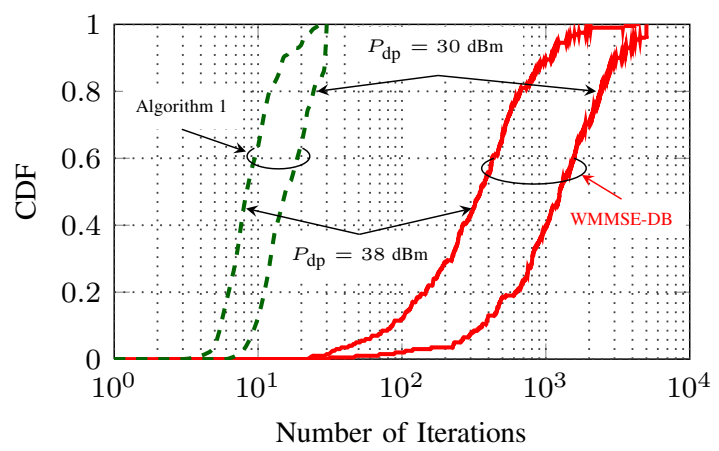

Fig. 3. Cumulative distribution of the numbers of iterations for 1000 channel realizations with $K_{b}=2$ and $N=4$.

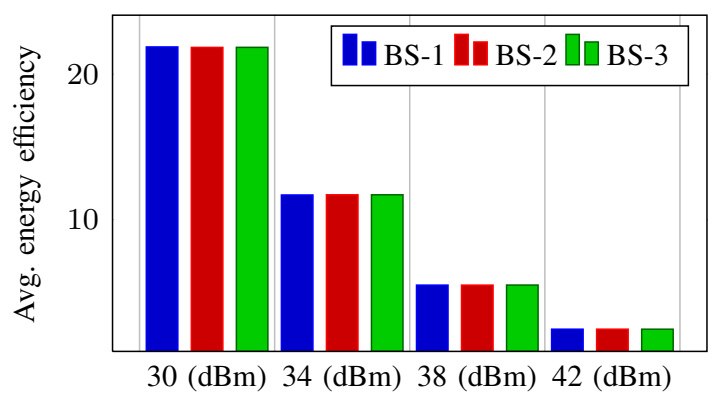

Fig. 4. Average energy efficiency versus $P_{\mathrm{dp}}$ with $K_{b}=2$ and $N=4$.

proposed design can maintain EE fairness among BSs.

\section{CONCLUSION}

We have studied the problem of maximizing the minimum EE among all BSs in multicell multiuser MISO downlink. The nonconvex optimization problem is approximated by a GCP in each iteration based on a framework of an inner approximation algorithm. In particular, we show that the problem at each iteration can be approximated as an SOCP which is much more efficiently solved using the modern conic solvers. The proposed algorithm outperforms the existing solutions in terms of the convergence rate and processing time, and is able to guarantee the EE fairness among all the BSs.

\section{REFERENCES}

[1] D. Feng, C. Jiang, G. Lim, J. Cimini, L. J., G. Feng, and G. Li, "A survey of energy-efficient wireless communications," IEEE Commun. Surveys Tuts., vol. 15, no. 1, pp. 167-178, Feb. 2013.

[2] B. Du, C. Pan, W. Zhang, and M. Chen, "Distributed energy-efficient power optimization for CoMP systems with max-min fairness," IEEE Commun. Lett., vol. 18, no. 6, pp. 999-1002, Jun. 2014.

[3] S. He, Y. Huang, S. Jin, F. Yu, and L. Yang, "Max-min energy efficient beamforming for multicell multiuser joint transmission systems," IEEE Commun. Lett., vol. 17, no. 10, pp. 1956-1959, Oct. 2013.

[4] A. Zappone, L. Sanguinetti, G. Bacci, E. Jorswieck, and M. Debbah, "Energy-efficient power control: A look at 5G wireless technologies," arXiv: $1503.04609 \mathrm{v} 1$.

[5] S. Schaible and J. Shi, "Recent developments in fractional programming: single ratio and max-min case," Nonlinear Analysis and Convex Analysis, pp. 493-505, 2004.

[6] B. R. Marks and G. P. Wright, "A general inner approximation algorithm for nonconvex mathematical programs," Operations Research, vol. 26, no. 4, pp. 681-683, Jul.-Aug. 1978.

[7] A. Ben-Tal and A. Nemirovski, "On the polyhedral approximations of the second-order cone," Mathematics of Operations Research, vol. 26, no. 2, pp. 193-205, May 2001.

[8] O. Arnold, F. Richter, G. Fettweis, and O. Blume, "Power consumption modeling of different base station types in heterogeneous cellular networks," in Proc.19th Future Network \& Mobile Summit (ICT Summit'10), Florence, Italy, Jun. 2010, pp. 1-8.

[9] J. Crouzeix and J. Ferland, "Algorithms for generalized fractional programming," Mathematical Programming, vol. 52, pp. 191-207, May 1991.

[10] R. Jagannathan, "On some properties of programming problems in parametric form pertaining to fractional programming," Management Science, vol. 12, no. 7, pp. 609-615, Mar. 1966.

[11] L.-N. Tran, M. F. Hanif, A. Tölli, and M. Juntti, "Fast converging algorithm for weighted sum rate maximization in multicell MISO downlink," IEEE Signal Process. Lett., vol. 19, no. 12, pp. 872-875, Dec. 2012.

[12] I. MOSEK ApS, 2014, [Online]. Available: www.mosek.com.

[13] A. Ben-Tal and A. Nemirovski, Lectures on modern convex optimization. Philadelphia: MPS-SIAM Series on Optimization, SIAM, 2001. 